\title{
Redes de Bragg utilizadas para mensuração da contração de polimerização de duas resinas acrílicas na moldagem aberta de prótese sobre implantes
}

\author{
- Moira Fatiga Universidade Federal do Paraná, Curitiba, PR, Brasil • Leandro Zen Karam Universidade Tecnológica \\ Federal do Paraná, Curitiba, PR, Brasil; Pontifícia Universidade Católica do Paraná, Curitiba, PR, Brasil • Nerildo Luiz \\ Ulbrich Universidade Federal do Paraná, Curitiba, PR, Brasil • Hypolito José Kalinowski Universidade Tecnológica Federal \\ do Paraná, Curitiba, PR, Brasil • Ana Paula Gebert de Oliveira Franco Pontifícia Universidade Católica do Paraná, Curitiba, \\ PR, Brasil; Universidade Tecnológica Federal do Paraná, Curitiba, PR, Brasil.
}

RESUMO | Objetivo: O objetivo do estudo foi comparar a contração máxima de polimerização e a variação máxima de temperatura de duas resinas acrílicas utilizadas na união dos postes de moldagem por meio de sensores de redes de Bragg. Material e Métodos: Dois implantes Cone Morse (Neodent) foram inseridos em um osso artificial. Sobre os implantes foram adaptados dois postes de moldagem que foram unidos com fio dental e resina acrílica. Para o estudo foram selecionadas duas resinas acrílicas: Duralay e GC Pattern LS. Foram posicionados dois sensores de fibra ótica: um em contato com a resina e outro no interior de uma agulha. As deformações máximas ( $\mu$ Strain) e a variação de temperatura máxima $\left({ }^{\circ} \mathrm{C}\right)$ foram medidas durante 34 minutos $(\mathrm{N}=28)$. Os dados foram analisados por meio da ANOVA a dois critérios e teste de múltiplas comparações de Games Howell ( $<$ o,05). Resultados: A Duralay apresentou maior contração de polimerização que a GC Pattern LS. O tempo não influenciou nos valores de contração de polimerização, mas influenciou na variação de temperatura máxima. Conclusão: A resina acrílica GC Pattern LS revelou menores valores de contração máxima de polimerização que a resina Duralay. Em contrapartida, A Duralay apresentou maiores valores de temperatura que a GC Pattern LS.

DESCRITORES | Resina; Contração; Polimerização; Temperatura; Moldagem; Implantodontia.

ABSTRACT | Bragg gratings used to measure the shrinkage polymerization of two acrylic resins in opened molding prosthesis on implants - Objective: The aim of this study was to compare the maximum polymerization shrinkage and maximum temperature variation of two acrylic resins used to join transfer copings through Bragg's grating sensors. Method: Two Morse Taper (Neodent) implants were inserted into an artificial bone (Sawbones), and two transfer copings were screwed at the implantsand joint with dental floss and acrylic resin. Two acrylic resins were selected for the study: Duralay and GC Pattern LS. Two fiber optical sensors were placed, one in contact with the resin and another inside a needle. The maximum deformation $\left(\mu\right.$ Strain) and the maximum temperature variation $\left({ }^{\circ} \mathrm{C}\right)$ were measured during 34 minutes $(\mathrm{N}=28)$. Data were analyzed using two way ANOVA and Games Howell multiple comparisons test ( $\mathrm{p}$ 0.05). Results: Duralay showed higher polymerization shrinkage than the GC Pattern LS. Time did not influence the polymerization shrinkage values, but influenced the maximum temperature variation. Conclusion: The acrylic resin GC Pattern LS revealed lower maximum shrinkage values than Duralay, and Duralay presented higher maximum temperature values than GC Pattern LS.

DESCRIPTORS | Resin; Shrinkage; Polymerization; Temperature; Impression; Implantology.

AUtORA CORRESPONDENTE | Ana Paula Gebert de Oliveira Franco Universidade Tecnológica Federal do Paraná • Rua Niccolo Paganini, 415, Vista Alegre Curitiba, PR, Brasil • 80820-180 E-mail: anapaula.gebert@gmail.com

- Received May 03,2015 • Accepted Jun 15, 2015

- Dol http://dx.doi.org/10.11606/issn.2357-8041.clrd.2015.118419 


\section{INTRODUÇÃO}

Os implantes dentários foram criados no ano de 1969 para reabilitar de forma funcional e estética dentes perdidos devido a cáries extensas, fraturas e problemas periodontais, sendo aperfeiçoados, no que se refere à osseointegração, por Branemark nos anos 1990. ${ }^{1}$ Atualmente uma grande porcentagem das pessoas que apresentam perdas dentárias unitárias ou múltiplas optam pela reabilitação por meio da prótese sobre implantes.

A prótese sobre implantes deve ser construída de forma individual para cada paciente, de modo que o cirurgião-dentista deve utilizar componentes de moldagem e protéticos para essa finalidade. No caso de perdas dentárias múltiplas, preconiza-se a técnica da moldagem aberta, ${ }^{2}$ na qual são utilizados postes de moldagem unidos com fio dental e resina acrílica, com o objetivo de determinar a passividade dos componentes, ou seja, que os postes de moldagem não se movam durante a inserção e remoção da moldeira. Essa esplintagem facilita que o técnico em prótese possa obter o encaixe preciso do poste de moldagem à plataforma do implante, que pode ser de formato triangular, quadrangular ou hexagonal. Caso os postes de moldagem não sejam unidos de forma rígida e estável, pode ocorrer desadaptação da coroa protética sobre o implante na boca do paciente.

As resinas acrílicas frequentemente utilizadas para unir os postes de moldagem são a Duralay (Reliance Dental MFG Company, Illinois, EUA) e a GC Pattern LS (GC America, Ilinois, EUA). A contração de polimerização e a exotermia são características inerentes às resinas acrílicas. ${ }^{3}$ Além de modificações na composição resina acrílica, a técnica de união dos postes de moldagem visa também reduzir a contração de polimerização. Nela os postes de moldagem são unidos, e após a polimerização inicial da resina (por volta de 15 minutos), a esplintagem é seccionada com um disco de carborundum ou ponta diamantada em alta rotação e a união é restabelecida com uma pequena porção da mesma resina acrílica. 4

Sensores de fibra ótica têm sido aplicados em estudos biomédicos em análises de materiais odontológicos, ${ }^{5}$ como cimentos resinosos ${ }^{6-8}$ e resinas acrílicas, ${ }^{9}$ e na validação de métodos de esterilização de materiais. Os sensores caracterizam-se pela capacidade de detecção de alterações térmicas e deformações mecânicas.

A contração de polimerização das resinas acrílicas utilizadas na união de postes de moldagem foi estudada por meio de medidores de tensão, ${ }^{10}$ dilatômetro 4 e microscópio reflex ${ }^{11}$, mas apresentam menor sensibilidade se comparados aos sensores de fibra ótica.

Os fabricantes da resina Pattern GC $\mathrm{LS}^{12}$ afirmam a menor contração de polimerização e exotermia desse material. Entretanto, não existem estudos que examinaram essas propriedades com equipamentos que apresentem elevada sensibilidade e as investiguem simultaneamente.

O objetivo do estudo foi comparar a contração máxima de polimerização e a variação máxima de temperatura de duas resinas acrílicas utilizadas na união dos postes de moldagem por meio de sensores de redes de Bragg.

\section{MATERIAL E MÉTODOS}

Foram selecionados dois implantes Cone Morse da marca Neodent (Curitiba, Brasil), com o diâmetro e comprimento de 4 e 15 milímetros, respectivamente.

Foi utilizado um osso artificial da marca Sawbones (Washington, EUA) que apresenta propriedades de módulo de elasticidade semelhantes aos ossos cortical e medular naturais. Nele foram demarcadas, com o auxílio de um modelo, as distâncias entre os implantes (17 mm), simulando a necessidade de uma prótese sobre múltiplos implantes. A localização dos pilares de implante foi 
referente aos dentes 44 e 46, com ausência do dente 45. Os implantes foram inseridos no osso artificial com perfurações escalonadas com brocas indicadas pelo fabricante, na velocidade de $600 \mathrm{rpm}$ em contra-ângulo redutor de velocidade, na proporção 20:1 (W\&H, Bürmoos, Áustria). Em seguida, também com o auxílio do contra-ângulo, em velocidade reduzida (250 rpm), com $5 \mathrm{~N}$. cm de torque final, eles foram aprofundados, ficando 1 milímetro abaixo da borda de perfuração.
Sobre os implantes, foram instalados os postes de moldagem (Neodent, Curitiba, Brasil) para a técnica aberta, unidos com fio dental e resina acrílica posteriormente.

No presente estudo foram selecionadas duas resinas acrílicas mais comumente utilizadas para unir postes de moldagem na implantodontia. As composições das resinas acrílicas utilizadas estão dispostas na Tabela 1.

Tabela 1 | Composição das resinas acrílicas utilizadas no estudo.

\begin{tabular}{c|c|c|c|}
\hline Resina acrílica & Tipo & Composição & Fabricante \\
\hline Duralay & Metacrilato & $\begin{array}{r}\text { Copolímero de metacrilato plastificável, monômero } \\
\text { de metacrilato, parafina, óleo mineral. }\end{array}$ & $\begin{array}{r}\text { Reliance Dental Mfg } \\
\text { Company, Ilinois, EUA. }\end{array}$ \\
\hline GC Pattern LS & Metacrilato & Polimetilmetacrilato, polietilmetacrilato, peróxido & GC América, Ilinois, EUA. \\
\hline
\end{tabular}

O pó e o líquido das resinas acrílicas foram pesados em uma balança analítica de precisão AUW220D (Shimadzu, América do Norte) para padronizar a quantidade de resinas acrílicas utilizadas em cada esplintagem. Para ambas as resinas acrílicas, foram utilizadas 0,14 g de pó e 0,05 g de líquido, que foram aplicados com um pincel de pelo de Marta fino utilizando a técnica de Nealon, com a qual se molha o pincel no monômero e, em seguida, no copolímero da resina acrílica, para então ser levado em contato com o fio dental. Foi também padronizada a quantidade de dez pinceladas, sendo três para cada poste de moldagem e quatro sobre o fio dental entre os postes de moldagem.

Foram utilizados dois sensores de fibra ótica, sendo um deles posicionado próximo ao centro da esplintagem em contato direto com a resina acrílica; e outro posicionado no interior de uma agulha hipodérmica de $0,4 \mathrm{~mm}$ de diâmetro. $\mathrm{O}$ sensor em contato com a resina acrílica é sensível às deformações e às variações de temperatura. $\mathrm{O}$ sensor posicionado no interior da agulha é sensível apenas às variações de temperatura. Dessa forma pode-se obter separadamente os valores das duas variáveis.

Os sensores iniciaram a medição logo após a colocação da resina acrílica (1 minuto em média) entre os postes de moldagem. O tempo total de medição foi de 34 minutos, polimerizando a barra como um todo, sem seccioná-la e reuni-la.

Os valores de contração máxima de polimerização ( $\mu$ Strain) e variação térmica máxima $\left({ }^{\circ} \mathrm{C}\right)$ foram obtidos em dois intervalos definidos: tempo 1 (T1) - de o a 17 minutos; e tempo 2 (T2) - de 17 a 34 minutos. Para análise estatística, foram utilizados os testes de normalidade de Kolmogorov-Smirnov, de homogeneidade de variância, com aplicação de ANOVA a dois critérios e múltiplas comparações de Games Howell $(\mathrm{p}<\mathrm{o}, \mathrm{05})$. 


\section{RESULTADOS}

A aplicação de ANOVA a dois fatores para a variável dependente "contração máxima” ( $\mu$ Strain), segundo a resina, apresentou valor de $\mathrm{F}$ igual a 120,57, que foi estatisticamente significante, uma vez que $\mathrm{p}<0,05$, indicando existir diferença entre os valores médios dessa variável, independente de tempo (Tabela 2).
O mesmo procedimento, porém segundo o fator tempo, apresentou valor de $\mathrm{F}$ igual a 1,70, que foi estatisticamente não significante, uma vez que $\mathrm{p}>$ 0,05, indicando não existir diferença entre os valores médios da variável dependente "contração máxima” ( $\mu$ Strain) segundo tempo, independente de resina (Tabela 3).

Tabela 2 | Estatística descritiva de contração máxima de polimerização das resinas acrílicas utilizadas no estudo.

\begin{tabular}{c|c|c|c}
\hline Resina acrílica & Média $(\mu$ Strain $)$ & Desvio padrão & N \\
\hline Duralay & $-349,31^{\text {a }}$ & 109,4 & 28 \\
\hline GC Pattern LS & $-99,84^{\text {b }}$ & 54,02 & 28 \\
\hline
\end{tabular}

* Letras minúsculas diferentes revelam diferenças estatísticas significativas na coluna $(p<0,05)$.

Tabela 3 || Estatística descritiva dos valores de contração máxima de segundo o tempo.

\begin{tabular}{c|c|c|c}
\hline Tempo & Média $(\mu$ Strain) & Desvio padrão & N \\
\hline T1 & $-209,77^{a}$ & 135,77 & 28 \\
\hline T2 & $-239,38^{a}$ & 168,12 & 28 \\
\hline
\end{tabular}

* Letras minúsculas diferentes revelam diferenças estatísticas significativas na coluna $(p<0,05)$.

Com relação à interação entre resina $\times$ tempo, para a variável "contração máxima" ( $\mu$ Strain), o valor de F foi de 1,93, mostrando-se estatisticamente não significante e indicando não existir interação entre resina $\times$ tempo $(\mathrm{p}>0,05)$. Para os dois tempos avaliados, a resina GC Pattern LS apresentou contração estatisticamente inferior à resina Duralay. Além disso, não houve diferenças estatisticamente significantes entre os tempos para um mesmo material (Tabela 4).

Tabela 4 | Estatística descritiva da contração máxima referente à interação resina x tempo.

\begin{tabular}{c|c|c|c}
\hline Resina acrílica/tempo & Média $(\mu$ Strain $)$ & Desvio padrão & N \\
\hline Duralay/T1 & $-318,73^{\text {a }}$ & 99,93 & 14 \\
\hline Duralay/T2 & $-379,89^{\text {a }}$ & 113,38 & 14 \\
\hline GC Pattern LS/T1 & $-100,8^{\text {b }}$ & 52,2 & 14 \\
\hline GC Pattern LS/T2 & $-98,88^{\text {b }}$ & 57,74 & 14 \\
\hline
\end{tabular}

* Letras minúsculas diferentes revelam diferenças estatísticas significativas na coluna $(p<0,05)$. 
No Gráfico 1 pode-se observar a curva média da contração de polimerização das resinas estudadas no tempo total de o a 34 minutos.

Nesse experimento, as amostras de resina Duralay mostraram uma elevação acentuada da temperatura: o valor médio atingiu $43^{\circ} \mathrm{C}$ durante os primeiros três minutos. A resina GC Pattern LS demonstrou uma elevação de temperatura média de $16^{\circ} \mathrm{C}$ durante os dez minutos iniciais (Tabela 5).

Gráfico 1 | Curva média da Contração de polimerização das resinas acrílicas estudadas.

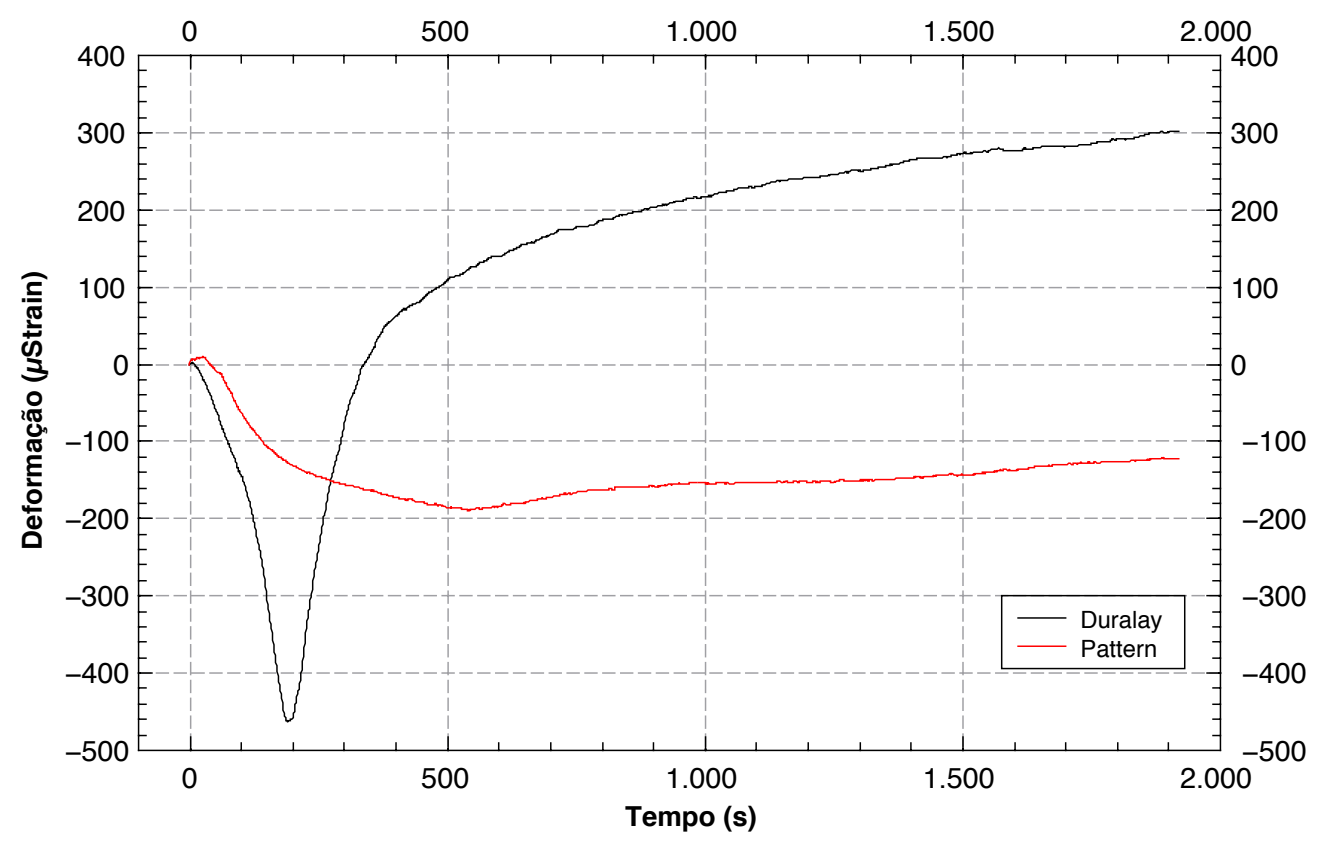

Tabela 5 | Estatística descritiva da variação máxima de temperatura $\left({ }^{\circ} \mathrm{C}\right)$ segundo Duralay e GC Pattern LS, e Tempo 1 (T1) e Tempo 2 (T2).

\begin{tabular}{|c|c|c|}
\hline Resina $\times$ tempo & Média $\left({ }^{\circ} \mathrm{C}\right)$ & Desvio padrão \\
\hline Duralay/T1 & $43,43^{a}$ & 4,94 \\
\hline Duralay/T2 & $5,45^{b}$ & 3,35 \\
\hline GC Pattern LS/T1 & $15,88^{c}$ & 0,91 \\
\hline GC Pattern LS/T2 & $16,24^{c}$ & 0,93 \\
\hline
\end{tabular}

* Letras minúsculas diferentes revelam diferenças estatísticas significativas na coluna $(p<0,05)$.

O aumento da temperatura inicial observado para a Duralay é seguido por uma diminuição e estabilização semelhante. A curva de resina GC
Pattern LS mostrou apenas uma pequena redução na temperatura seguida por uma estabilização (Gráfico 2). 
Gráfico 2 || Curva média da variação de temperatura das resinas acrílicas estudadas.

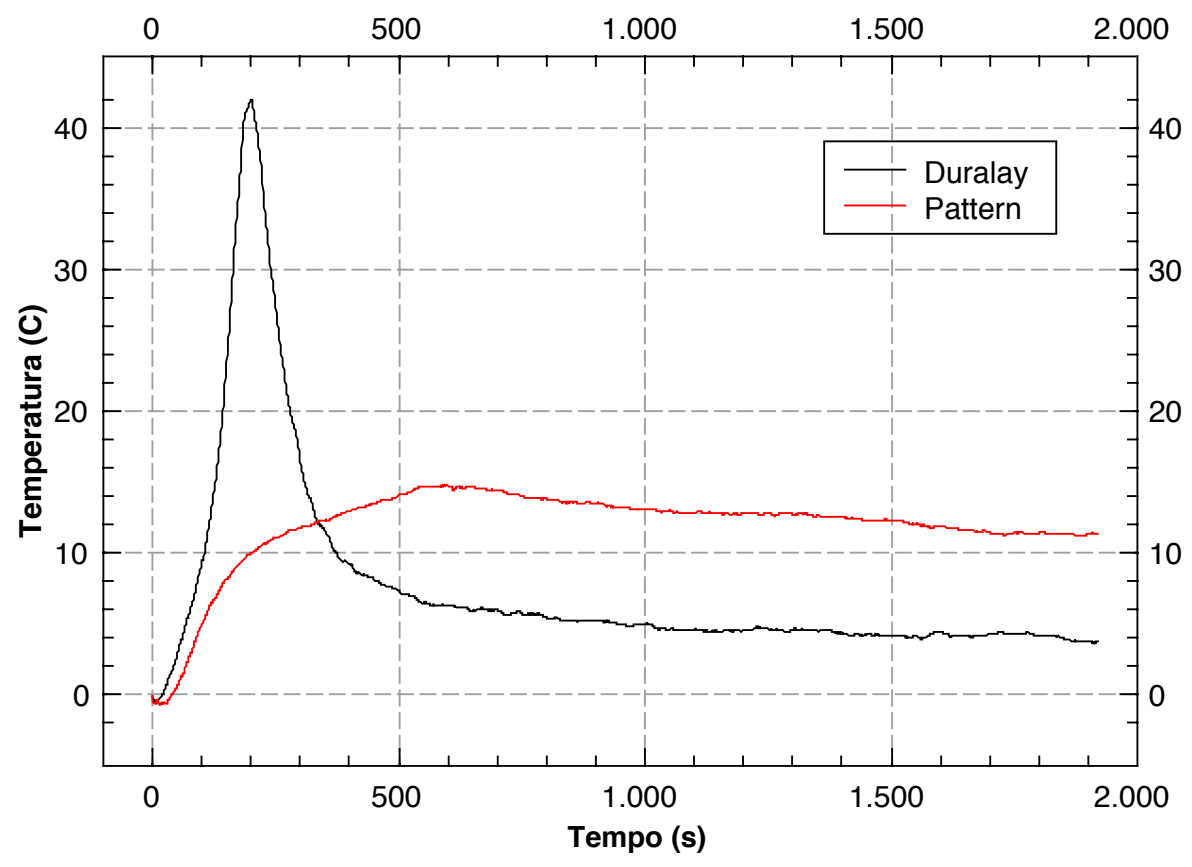

\section{DISCUSSÃO}

A contração de polimerização nas resinas acrílicas é um fenômeno que ocorre devido à quebra da dupla ligação vinílica pelo radical livre e, consequentemente, união do monômero à cadeia em crescimento através de uma ligação covalente.

Li et al. ${ }^{13}$ relataram que as resinas acrílicas compostas por MMA/HEMA contêm grupos funcionais sol-gel (sol é uma dispersão de partículas coloidais estável em um fluido, enquanto gel é um sistema formado pela estrutura rígida de partículas coloidais - gel coloidal ou de cadeias poliméricas - gel polimérico que imobiliza a fase líquida nos seus interstícios) que reduzem a contração de polimerização. No presente estudo observou-se que a GC Pattern LS demonstrou menores valores de contração máxima de polimerização que a Duralay, estando de acordo com os achados desses autores, ${ }^{13} \mathrm{de}$ Cerqueira et al..$^{10} \mathrm{e}$ com as afirmações do fabricante da resina GC Pattern LS. ${ }^{12} \mathrm{O}$ fenômeno de contração da Duralay foi seguido de uma expansão, enquanto a GC Pattern LS demonstrou uma tendência à estabilização da curva. $\mathrm{O}$ estudo de Gibbs et al. ${ }^{14}$ mostrou não haver diferenças significativas para a contração volumétrica entre Duralay 5,72\% $(0,89)$ e GC Pattern de 5,07\% (1,36).

Mojon et al. ${ }^{4}$ demonstraram que a contração de polimerização total da resina acrílica Duralay foi de $7 \%$ a $9 \%$ e que a contração máxima ocorreu nos primeiros 17 minutos à temperatura ambiente. Por esse motivo, o presente estudo comparou as diferenças de contração máxima de polimerização nos primeiros 17 minutos (tempo 1) e de 17 a 34 minutos (tempo 2). Os resultados do estudo não demonstraram diferenças estatísticas significativas para a deformação máxima segundo o tempo.

Após os 17 minutos iniciais de presa, recomenda-se cortar o centro da esplintagem e reconectá-la com a mesma resina acrílica, com o objetivo de reduzir a contração de polimerização. Estudos como o de Franco et al. ${ }^{9}$, Cerqueira et al. ${ }^{10}$ e Lee et al ${ }^{15}$ revelaram que, após esse procedimento, a contração dos materiais resinosos acrílicos realmente se reduziu, além de menores distorções serem obtidas, se comparada 
à técnica que apenas conecta os postes de moldagem. Apesar do conhecimento dos dados mencionados, o intuito do presente estudo foi comparar a contração de polimerização das resinas acrílicas Duralay e GC Pattern LS, e não a técnica de esplintagem.

Além da contração de polimerização, as resinas acrílicas investigadas geram uma significante energia térmica durante a reação de presa. Quando essas resinas são aplicadas diretamente sobre os postes de moldagem, elas podem transferir calor para os tecidos adjacentes, danificando a interface osso-implante. ${ }^{16} \mathrm{O}$ efeito do superaquecimento dos tecidos nessa interface pode causar estase vascular, necrose irreversível ou reabsorção do tecido ósseo e comprometer a capacidade de diferenciação óssea. ${ }^{17,18}$

O presente estudo revelou o valor médio para a máxima alteração de temperatura de $43,4^{\circ} \mathrm{C}$ para a Duralay no tempo 1 e de $5,5^{\circ} \mathrm{C}$ para o tempo 2. A redução dos valores de temperatura no tempo 2 pode ser explicada pela menor mobilidade das cadeias carbônicas nessa fase, se comparada à fase inicial da polimerização. Os valores referentes ao pico de temperatura da Duralay de $50^{\circ} \mathrm{C}$ obtidos no presente estudo estão de acordo com os estudos de Ko et al. ${ }^{19}$ e de Tapia Silva et al., ${ }^{20}$ que demonstraram $56,7^{\circ} \mathrm{C}$ em 7,5 minutos, utilizando um termopar ligado a um termômetro digital.

A GC Pattern LS mostrou variação média de temperatura máxima de $18,1^{\circ} \mathrm{C}$ para o tempo 1 e $16,2^{\circ} \mathrm{C}$ para o tempo 2 . Os valores de variação térmica mostraram-se mais elevados na resina Duralay quando comparada à GC Pattern LS e no tempo 1, se comparado ao tempo 2. A Duralay revelou elevados valores térmicos que podem danificar os tecidos adjacentes, porém pode-se observar que no tempo 2 ela mostrou maior redução dessa variável quando comparada à GC Pattern LS. Considerando a variável temperatura, pode-se afirmar que a resina acrílica GC Pattern LS pode ser indicada para uma utilização mais generalizada, por apresentar menores riscos biológicos.
Futuros estudos devem investigar a transferência de calor dos postes de moldagem para as estruturas biológicas adjacentes.

\section{CONCLUSÃO}

Considerando as limitações do presente estudo, pode-se concluir que:

- A resina acrílica GC Pattern LS revelou menores valores de contração máxima de polimerização que a resina Duralay. Isso revela maior passividade da GC Pattern LS em moldagens abertas;

- A variação da temperatura máxima foi maior para a resina acrílica Duralay;

- O tempo de polimerização não influenciou nos valores de contração de polimerização;

- A variação de temperatura máxima foi menor para o tempo 2 em ambas as resinas acrílicas.

\section{REFERÊNCIAS}

1. Branemark PI, Zarb GA, Albrektsson T. Tisue-integrated prosthesis: osseointegration in clinical dentistry. J Prosthet Dent. 1985;54(4):611-2. doi: http://dx.doi.org/10.1016/oo223913(85)90460-3.

2. Carr AB, Sokol J. Accuracy of casts produced by the Nobelpharma impression techniques. J Dent Res. 1991;70(special issue):290.

3. Skiner EW. Acrylic resins: an appraisal of their use in dentistry. J Am Dent Assoc. 1949;39(3):261-8. doi: http://dx.doi. org/10.14219/jada.archive.1949.0217.

4. Mojon P, Oberholzer JP, Meyer JM, Belser UC. Polymerization shrinkage of index and pattern acrylic resins. J Prosthet Dent. 1990;64(6):684-8. doi: http://dx.doi.org/10.1016/oo223913(90)90296-O.

5. Milczewski MS, Silva JCC, Abe I, Carvalho LM, Fernandes RC, Kalinowski HJ, et al. FBG application in the determination of setting expansion of dental materials. Meas Sci Technol. 2006;17(5):1152-6. doi: 10.1117/12.623633.

6. Milczewski MS, Silva JCC, Paterno AS, Kuller F, Kalinowski HJ. Measurement of composite shrinkage using a fibre optic Bragg grating sensor. J Biomat Sci Polym Edn. 2007;18(4):383-92. doi: 10.1163/156856207780425004. 
7. Antilla EJ, Krintilä OH, Laurila TK, Lassila LV, Valittu PK, Hernberg RG. Evaluation of polymerization shrinkage and hydroscopic expansion of fiber-reinforced biocomposites using optical fiber Bragg grating sensors. Dent Mat. 2008;24(12):1720-7. doi: 10.1016/j.dental.2008.07.006.

8. Karam LZ, Franco APGO, Pulido CA, Pulido CA, Gomes OMM, Kalinowski HJ. In vitro and in situ fiber Bragg grating sensor analysis of two dental resin cements. J Lightwave Technol. 2015;33(12):2543-8. doi: 10.1109/ JLT.2014.2376182.

9. Franco APGO, Karam LZ, Galvão JR, Kalinowski HJ. Evaluation of shrinkage polymerization and temperature of diferent acrylic resins used to splinting transfer copings in indirect impression technique. In: The $24^{\text {th }}$ International Conference on Optical Fibre Sensors (OFS23); 2015, Curitiba. Published in SPIE Proceedings vol. 9634.

10. Cerqueira NM, Ozcan M, Gonçalves M, Rocha DM, Vasconcellos DK, Bottino MA, et al. A strain gauge analysis of microstrain induced by various splinting methods and acrylic resin types for implant impressions. Int J Oral Maxillofac Implants. 2012;27(2):341-5.

11. Herbst D, Nel JC, Driessen CH, Becker PJ. Evaluation of impression accuracy for osseointegrated implant supported superstructures. J Prosthet Dent. 2000;83(5):555-61.

12. GC Corporation. GC Pattern LS low shrinkage modelling: resin tips and tricks: technique and handling of GC pattern resin. 2009 [citado em 2016 dez 1]. Disponível em: http:// www.gcamerica.com/lab/products/PATTERN_RESIN_LS/ pattern_resin_tech_guide.pdf.
13. Li S, Shah A, Hsieh AJ, Haghighat R, Praveen SS, Mukherjee I, et al. Characterization of poly (2-hydroxyethylmethacrylate-silica) hybrid materials with silica contents. Polymer. 2007;48(14):3982-9. doi: http://dx.doi.org/10.1016/j.polymer.2007.05.025.

14. Gibbs SB, Versluis A, Tantbirojn D, Ahuja S. Comparison of polymerization shrinkage of pattern resins. J Prosthet Dent. 2014;112(2):293-8. doi: 10.1016/j.prosdent.2014.02.006.

15. Lee SJ, Cho SB. Accuracy of five implant impression technique effect of splinting material and methods. J Adv Prosthodont. 2011;3(4):177-85. doi: 10.4047/jap.2011.3.4.177.

16. Moulding MB, Teplitsky PE. Interpulpal temperature during direct fabrication of provisional restorations. Int J Prosthodont. 1990;3(3):293-304.

17. Eriksson RA, Albrektsson T, Grane B, McQueen D. Thermal injury to bone: a vital-microscopic description of heat effects. Int J Oral Surg. 1982;11(2):115-21.

18. Erikson RA, Albrektsson T. Assessment of bone viability after heat trauma: a histological, histochemical and vital microscopic study in the rabbit. Scand J Plast Reconstruct Surg. 1984;18(3):261-8.

19. Ko MJ, Pae A, Kim SH. In vitro study on exothermic reaction of polymer-based provisional crown and fixed partial denture materials measured by differential scanning calorimetry. $\mathrm{J}$ Kore Academ Prosthod. 2006;44(6):690-6.

20. Tapia Silva R, Valenzuela Aránguiz V, Zamorano Pino X, Baena Águila R. Cuantificatión de la generatión térmica en acrílicos de autopolimerización. Av Odontoestomatol. 2010;26(2):91-6. 